\title{
MECHANICAL DYNAMIC ANALYSIS OF THE LHC ARC CRYO-MAGNETS
}

\author{
K. Artoos, N. Bourcey, O. Calvet, O. Capatina, C. Hauviller, CERN, Geneva, Switzerland
}

\begin{abstract}
The arcs of the Large Hadron Collider (LHC) will contain around 1700 main superconducting dipoles and quadrupoles. The long and heavy magnets are placed on fragile composite support posts inside a cryostat to reduce the heat in-leak to the magnets super fluid helium bath. The presence of such fragile components like the support posts, the beam position monitors and the corrector magnets make the cryo-magnets very difficult to handle and transport. Furthermore, keeping the geometry of the cryo-magnets unchanged (in the range of $0.1 \mathrm{~mm}$ ) throughout the various transports and handling is essential for the good functioning of the future LHC.

A detailed dynamic analysis was performed to determine the behavior of the cryo-magnets under all the handling and transport conditions and to choose the related optimum parameters. The results of finite element modal calculations as well as experimental modal analyses are presented and compared. The maximum accelerations admissible during transport with several types of vehicle were computed. The accelerations experienced by both types of cryo-magnets were measured during real transport with different vehicles. The dynamic deformation of the support posts in the cryodipole was also measured. The methodologies of these analyses and their results are reported as well as the resulting specification for the transport during the LHC installation.
\end{abstract}

\section{INTRODUCTION}

The arcs of the LHC will contain a large amount of superconducting dipoles and quadrupoles. The arc dipoles installed into their cryostats, hereafter called cryo-dipoles, are $15 \mathrm{~m}$ long and have a mass of about 33 ton. The socalled short straight sections (SSS) include main quadrupoles together with their correctors and the beam position monitors. The SSS are about $6 \mathrm{~m}$ long and have a mass of about 8 ton.

All those cryo-magnets contain fragile components, such as the thin-walled support posts made of glass fiber reinforced epoxy, to reduce environmental heat in-leak to the magnets super fluid helium bath. The position of the magnet inside its cryostat (in the range of $0.1 \mathrm{~mm}$ ) as well as the integrity of all its components are compulsory factors for a good functioning of the future LHC.

The cryo-magnets dimensions and weight, the geometric constraints, as well as the fragile components, make them very difficult to handle and transport.

Understanding the cryo-magnets dynamic behavior is needed to avoid potential malfunctions provoked by vibrations during transport and handling.

\section{METHODOLOGY}

Important tools to assess the dynamic behavior of the cryo-magnets are numerical models of the cryo-dipole and the Short Straight Section. The models were first built with estimated parameters and then validated by measurements.

\section{Calculations}

The cryo-magnets were modeled with the finite element code Ansys ${ }^{\mathrm{TM}}$ (ref. [1], [2] and [3]). Components significant for the vibration behavior, i.e. cold mass, support posts, vacuum vessel and transport restraints, were detailed to a sufficient level.

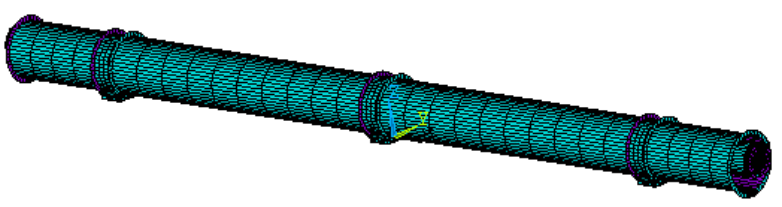

Figure 1: Cryo-dipole finite element model.

Several modal analyses were performed using the cryo-magnet finite element models together with different boundary conditions representing surface, road and tunnel handling and transport equipments.

\section{Measurements}

Two types of measurements were performed:

- modal analysis of cryo-magnets on concrete blocks

- cryo-magnet dynamic behavior during transport with different types of vehicles

The natural frequencies and the mode shapes of the cryo-magnets were determined with free (impact) and forced vibration. The harmonic and random forced excitation was applied by an electrodynamic shaker (see Figure 2) via an impedance head. Tri-axial PCB-ICPTM accelerometers measured the accelerations of the cryo-magnet components during the modal analysis.

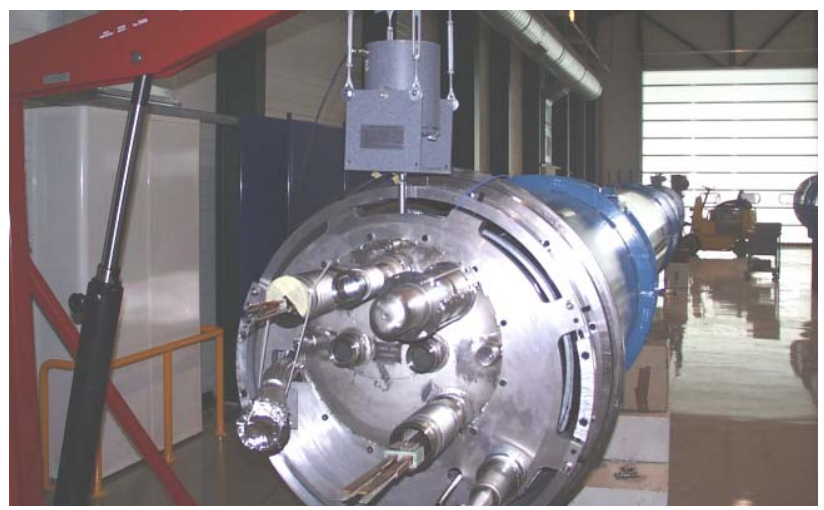

Figure 2: Cryo-dipole on concrete blocks modal analysis. 
During handling and transport, the same accelerometers were used together with LVDT gauges that measured the tri-axial dynamic deformations of the support posts.

Calculations and measurements were then combined to specify the maximum allowable accelerations during different types of handling and transport.

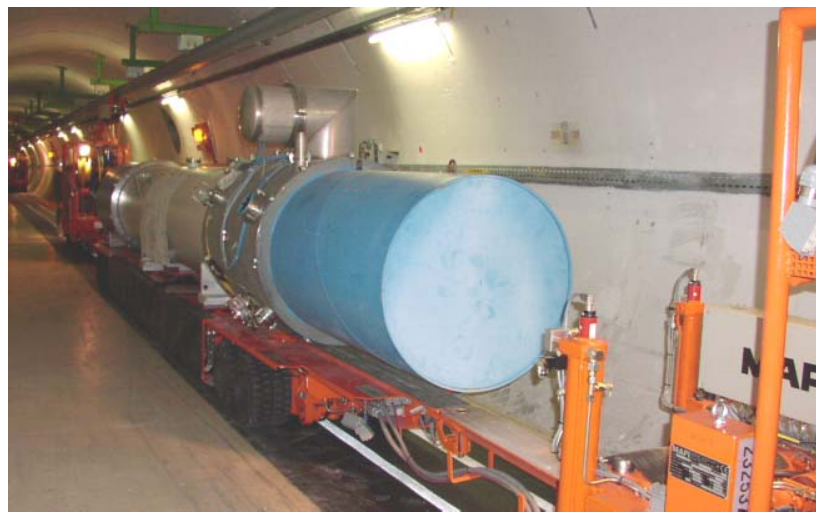

Figure 3: SSS on tunnel vehicle.

Figure 3 shows an acceleration and support post deformation test of a SSS during a transport in the LHC tunnel.

\section{RESULTS}

\section{Modal analysis}

Several cases were treated with the methods mentioned above. Experimental cryo-dipole and SSS modal analyses were performed. The natural modes were determined for three directions (lateral, vertical and longitudinal) up to $50 \mathrm{~Hz}$, with the cryo-magnets installed on concrete blocks (i.e. the boundary conditions during storage).

The natural frequencies and the modal shapes obtained permitted to validate the finite element models.

Table 1 gives an example of the comparison between tests and calculations, for the first four vertical modal shapes and natural frequencies of the cryo-dipoles installed on concrete blocks.

Table 1: Example of cryo-dipole on concrete blocks modal analysis results - comparison tests/calculations:

\begin{tabular}{|l|l|c|}
\hline \multirow{2}{*}{$\begin{array}{l}\text { Cryo-dipole vertical } \\
\text { modal shape }\end{array}$} & \multicolumn{2}{|c|}{ Frequency } \\
\cline { 2 - 3 } & Tests & Calculations \\
\hline & $8 \mathrm{~Hz}$ & $10 \mathrm{~Hz}$ \\
\hline & $16 \mathrm{~Hz}$ & $17 \mathrm{~Hz}$ \\
\hline & $28 \mathrm{~Hz}$ & $32 \mathrm{~Hz}$ \\
\hline & $36 \mathrm{~Hz}$ & $34.6 \mathrm{~Hz}$ \\
\hline
\end{tabular}

The complete modal analysis of cryo-dipole and SSS are reported in [2], [3], [4] and [6].

The validated finite element models were then used to compute the cryo-magnets behavior, when installed on various types of transport units. The new modal schemes were calculated for the cryo-magnets under transport conditions, by replacing the "concrete blocks" boundary conditions by appropriate new boundary conditions. For each type of boundary conditions, corresponding to a given transport vehicle, a new finite element modal analysis was performed.

As an example, the first four vertical modes of the cryo-dipole installed on a vehicle for road transport are presented in Table 2.

Table 2: Example of cryo-dipole on a road vehicle modal analysis results:

\begin{tabular}{|c|c|}
\hline Vertical modal shape & Calculated frequency \\
\hline \\
\hline
\end{tabular}

For each identified modal shape, the maximum admissible acceleration at a specific point of the magnet was calculated from the specified maximum deformations of the support posts. This specific point is the point where accelerometers will be installed during the transport.

\section{Behavior during transport}

Accelerations as well as support post deformations were measured during several cryo-magnet transports with surface, road and tunnel vehicles.

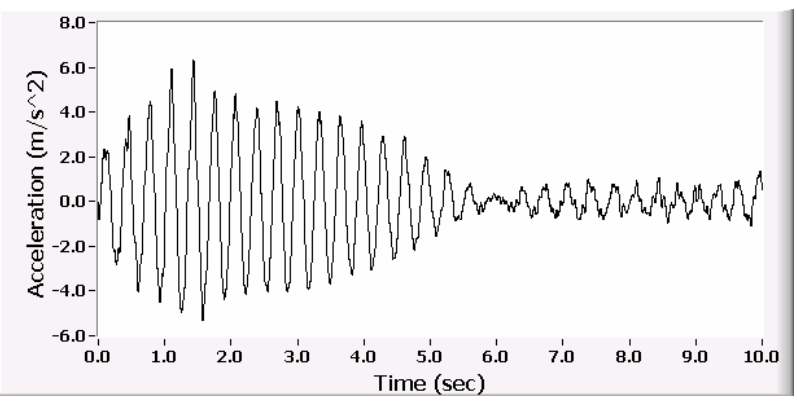

Figure 4: Event recorded in vertical direction during road transport of a cryo-dipole.

For the cryo-dipole road transport, the most important vibration amplitudes in the vertical direction were observed at a frequency of $3 \mathrm{~Hz}$, corresponding to the second mode identified in Table 2.

The modes with the most important amplitudes were identified for the three directions. For an exhaustive list, please refer to [4], [5] [6] and [7]. The measured accelerations and support post deformations were coherent. 
Knowing the modes that are excited during a given type of transport, and knowing the corresponding acceleration limits at a given point of the cryo-magnet, global accelerations limits were defined for each type of transport.

The accelerations measured at the extremity of the dipole cold mass and the support post deformations during road transport are presented in Table 3. They are compared with the maximum allowed values.

Table 3: Maximum admissible and measured accelerations for a cryo-dipole road transport

\begin{tabular}{|c|c|c|c|c|}
\hline & \multirow{3}{*}{$\begin{array}{c}\text { Lateral } \\
5\end{array}$} & \multirow{3}{*}{$\begin{array}{c}\text { Vertical } \\
7\end{array}$} & \multirow{3}{*}{\begin{tabular}{|c|} 
Longitudinal \\
4
\end{tabular}} \\
\hline & & & & \\
\hline$\stackrel{\mathscr{0}}{0}$ & Limits & & & \\
\hline 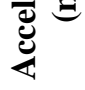 & Tested & 3.2 & 6.5 & 1.1 \\
\hline \multirow{2}{*}{ ڤ气 } & Limits & 0.42 & 0.16 & 0.42 \\
\hline & Tested & 0.05 & 0.12 & 0.05 \\
\hline
\end{tabular}

Table 3 shows measured values in the vertical direction close to the admissible limits.

\section{SERIAL TRANSPORT}

More than 1200 cryo-dipoles and more than 350 SSS will have to be transported and handled several times, and with different vehicles and handling devices, during the installation phase of the LHC. All these operations are critical. Table 3 indicates that the measured values in the vertical direction, during cryo-dipole road transport, are close to the limits.

A geometry and integrity check of the cryo-magnets is not possible in the tunnel, prior to installation. Localizing and removing a damaged cryo-magnet installed in the tunnel, would be an important time-consuming operation. A detailed qualification of all the vehicles and handling devices that will be used during the LHC installation is hence needed. Each transported and handled cryo-magnet will also be monitored to ensure that the acceleration limits presented in the precedent paragraph have not been exceeded. A tri-axial acceleration-monitoring device will be placed at a specified position on each transported cryo-magnet.

\section{CONCLUSIONS}

A detailed dynamic analysis was performed to determine the behavior of the cryo-magnets under all the handling and transport conditions.

Finite element calculations and experimental modal analyses of cryo-magnets under storage conditions (placed on concrete blocks) were performed up to $50 \mathrm{~Hz}$. The calculations and test results were compared and the finite element models were validated.
The finite element models were then used to calculate the modal scheme of the cryo-magnets under various transport and handling conditions. The dynamic behavior of the cryo-magnets during handling and transport was also tested, and energetic modes were identified. From both, calculations and test results, the maximum accelerations admissible during transport with several types of vehicles were specified.

The accelerations experienced by both types of cryo-magnets were measured during real transport with surface, road and tunnel vehicles. The dynamic deformations of the support posts were also measured. Accelerations and dynamic deformations were coherent.

The tests have shown that it is possible to handle and transport the cryo-magnets without damage. Several measured accelerations were however very close to the limits. Each transported and handled cryo-magnet must hence be monitored to insure that the specified acceleration limits have not been exceeded, to avoid installation of damaged cryo-magnets.

\section{REFERENCES}

[1] https://edms.cern.ch/file/332842/1.0/TN02_11.pdf, O. Calvet, C. Hauviller; "Analyse du comportement mecanique des cryodipoles du LHC - 1ere partie: Statique"; LHC-CRI Technical Note 2002-11

[2] O. Calvet, C. Hauviller; "Analyse du comportement mecanique des cryodipoles du LHC - 2ème partie: Dynamique"; LHC-CRI Technical Note 2002-12

[3] https://edms.cern.ch/file/352974/1/2002-003.pdf, P. Cupial, J. Snamina; "The verification of the computational models of the LHC Short Straight Section"; Technical Note EST-ME/2002-003

[4] https://edms.cern.ch/file/348871/2/TN02_07.pdf, K. Artoos, O. Calvet, O. Capatina; "Experimental modal analysis and acceleration measurements during surface transport of a LHC cryodipole"; LHCCRI Technical Note 2002-07

[5] https://edms.cern.ch/file/350433/1.0/TN02-13.pdf, K. Artoos, N. Bourcey, O. Capatina; "Acceleration and support posts deformation measurements during surface transport of a LHC cryodipole", LHC-CRI Technical Note 2002-13

[6] https://edms.cern.ch/file/347269/2.1/TN02_06.pdf, K. Artoos, O. Capatina; "Experimental modal analysis and acceleration measurements during transport of a LHC Short Straight Section", LHCCRI Technical Note 2002-06

[7] K. Artoos, O. Capatina, "Acceleration and support posts deformation measurements during surface and tunnel transport of a LHC Short Straight Section"- to be published 